\title{
Dystextia: An Early Sign of Pregnancy-Associated Meningioma
}

\author{
James B. Hannah'1, Phillip Kissel'2, Bianca Russell ${ }^{3}$, Jo Ellen Hose ${ }^{4}$ \\ ${ }^{1}$ Central Coast Pathology Consultants, San Luis Obispo, USA \\ ${ }^{2}$ Department of Neurosurgery, Sierra Vista Regional Medical Center, San Luis Obispo, USA \\ ${ }^{3}$ Cincinnati Children's Hospital, Cincinnati, USA \\ ${ }^{4}$ California Polytechnic State University, San Luis Obispo, USA \\ Email: jhannah@ccpathology.com
}

Received 20 February 2014; revised 20 March 2014; accepted 28 March 2014

Copyright (C) 2014 by authors and Scientific Research Publishing Inc.

This work is licensed under the Creative Commons Attribution International License (CC BY). http://creativecommons.org/licenses/by/4.0/

(c) (i) Open Access

\begin{abstract}
Objective: The authors report a case of meningioma causing incomprehensible and excessive text messaging in a postpartum woman. Case Report: We report the case of expressive dystextia related to a postpartum, progesterone receptor-positive meningioma. Growth of meningiomas can accelerate during pregnancy and the postpartum period due to expression of hormone receptors, particularly progesterone. This is the first study describing dystextia related to a brain tumor; previous cases of dystextia are associated with stroke and complex migraine. Here expressive dystextia, the inability to compose syntactically comprehensible text messages, preceded acute neurologic signs by several months, and surgical resection of the meningioma eliminated all neurologic sequelae. Possible genetic etiologies for meningioma are discussed since this patient's prior thyroid cancer at age 18 suggests a relationship between the two neoplasms. Conclusions: Since text messaging is becoming one of the principal forms of communication in our society and requires both cognitive and motor skills, clinicians should be aware that dystextia may be the initial sign of significant neurologic pathology. We propose that an inquiry about altered text messaging frequency and comprehensibility should be a standard part of the neurologic evaluation.
\end{abstract}

\section{Keywords}

Meningioma, Brain Tumor, Dystextia, Progesterone Receptor, Text Messaging

\section{Introduction}

Dystextia, the difficulty or inability to send text messages, has recently been described as a resolvable symptom of ischemic stroke [1]-[3] and complicated migraine [4]. Since texting is a complicated activity involving vision, 
language and motor skills, dystextia is expected to become an increasingly common sign of neurologic disease with growing cell phone communications [1] [4]. Neurologic deficits can affect motor skills essential for typing text messages [1] or produce the text messaging equivalent of expressive dysphasia [2]-[4] which we term "expressive dystextia" and define as the loss of ability to compose syntactically comprehensible text messages. To our knowledge, we report the first case of expressive dystextia as an early sign of pregnancy-associated meningioma.

Meningiomas are the most frequently diagnosed primary brain tumor and have been routinely recognized in the context of pregnancy and the postpartum period [5]-[10]. Pregnancy may contribute to rapid expansion of normally slow-growing cerebral meningiomas due to hormone receptor expression [8] [11] [12]. Approximately half of benign intracranial meningiomas are progesterone receptor-positive, and the increase in serum progesterone levels during the second half of pregnancy may fuel growth of these low-grade neoplasms [13] [14]. Meningiomal expansion has also been reported with increased progesterone production during the luteal phase of the menstrual cycle and in a male-to-female transsexual patient receiving estrogen-progestin therapy [8] [15]. In one patient, reduction of multiple meningiomas and complete resolution of one tumor were accomplished by discontinuation of the long-term use of a progesterone agonist [16]. The prevalence of meningiomas found incidentally in older women is at least $1 \%$, and two systematic reviews document a higher meningioma risk for postmenopausal women currently receiving hormone replacement therapy [5] [7] [10] [17]. However, the effect of routine oral contraceptive use on meningioma risk is unclear except for an increased risk of using long-acting hormonal contraceptives [7] [10].

Patients with meningiomas may exhibit evolving subtle language and speech deficits or may present with acute neurological changes in mental status [8] [9] [18] [19]. This case report adds expressive dystextia to the neurologic deficits associated with meningioma. With the worldwide increase in text communication, difficulty in text messaging may become a more widely appreciated sign of incipient neurologic disease [1].

\section{Case Report}

\subsection{History and Presentation}

The patient, a 36-year-old right-handed female, was admitted to the emergency room after being found with a depressed level of consciousness and difficulty with arousal. She was 7 months postpartum with a 4-month history of depression, decreasing cognition, and increasing headache. Prior to the birth of her child, she sent text messages to her relatives once or twice weekly. As her depression worsened, the frequency of her text messaging increased to approximately two every hour, day and night, sometimes to people in the next room. Relatives noted progressive incomprehensibility of her text communications during this period. Previous history included surgery for thyroid cancer at age 18 followed by continuous pharmacologic treatment for hypothyroidism but no radiation therapy to head or neck. Informed consent was obtained from the patient for both presentation and publication of her case.

\subsection{Treatment}

An unenhanced computed tomography (CT) scan of the brain from the emergency room showed a $7.8 \mathrm{~cm}$ right frontal mass with acute intratumoral hemorrhage and severe shift of midline (Figure 1). An emergent right frontal craniotomy was performed. The darkened dura was opened, revealing a bluish underlying cerebral cortex and a hematoma spontaneously extruding through the pial layer. Intraoperatively, the lesion was relatively sharply demarcated and was dural-based. The tumor had a pseudocapsule which allowed separation from the surrounding edematous cortex. The dural attachment was localized to the posterior wall of the right frontal sinus. There was no evidence of macroscopic invasion of the pia, pericranium or inner table of the bone. The craniotomy closure was routine but multiple blood products were transfused during the procedure. A post-operative CT scan confirmed gross total resection of the brain tumor with expected, residual right hemispheric swelling (Figure 2). Post-operative management for a benign meningioma with a gross total resection is surveillance. This includes observation for potential neurological decline and a yearly MRI scan with contrast.

\subsection{Pathological Findings}

Examination of formalin-fixed intraoperative tissue revealed a meningioma, World Health Organization (WHO) 

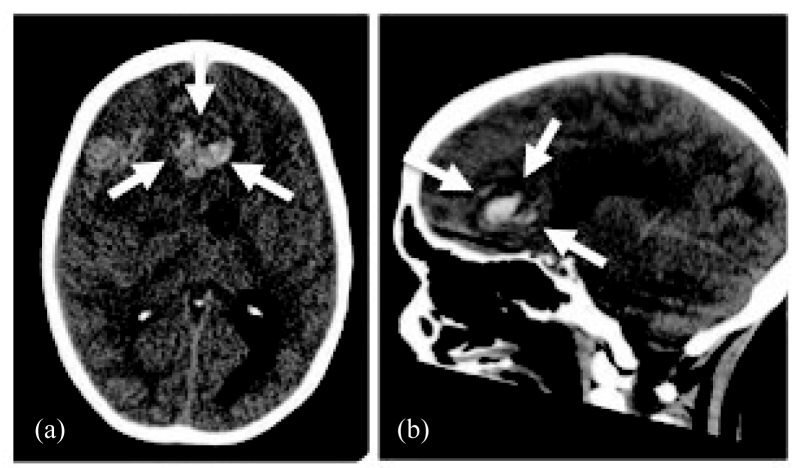

Figure 1. Pre-operative axial (a) and sagittal (b) non-contrast CT scans of large right frontal meningioma (arrows) with hematoma.
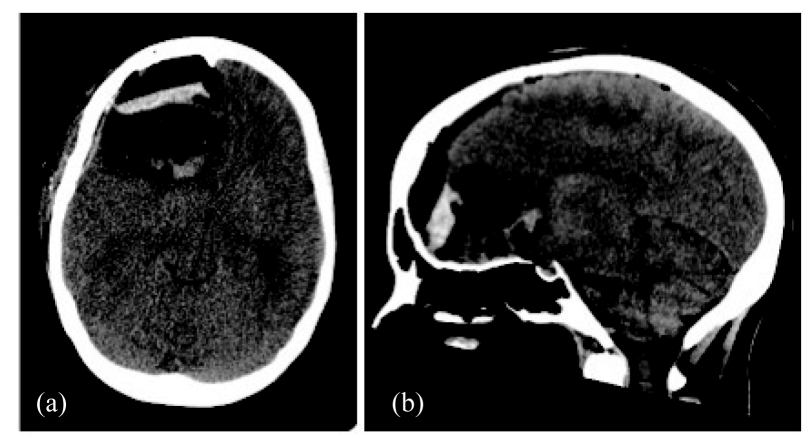

Figure 2. Post-operative axial (a) and sagittal (b) noncontrast CT scans of resected right frontal meningioma.

Grade 1. Routine hematoxylin and eosin sections demonstrated a meningothelial neoplasm with areas of clear cell change and loss of whirling architecture (Figure 3). No glial invasion or obvious tumor necrosis were identified, and the specimen exhibited no mitotic figures or cytologic atypia. Results of well-controlled immunohistochemical stains were: glial fibrillary acidic protein (GFAP)-negative, thyroid transcription factor-1 (TTF-1)negative, and cytokeratin CAM5.2-negative with weak vimentin and strong positive epithelial membrane antigen (EMA) nuclear staining. The Ki-67 proliferative index was approximately 5\%. Tumor cells were estrogen receptor-negative and highly positive for progesterone receptors with $95 \%$ of the cells exhibiting moderate to strong nuclear staining (Figure 4).

\subsection{Outcome}

The neurologic assessment on day 2 post-operative revealed mild to moderate attention and short-term memory deficits. Ability to swallow and ambulate improved until her discharge to family on day 4 . She had minimal residual expressive dysphasia that resolved, and she normalized her speech function within three weeks. She returned to all normal mental and physical activities by the third post-operative month. Her family reported that she had resumed coherent daily texting activities, and she was not contemplating further pregnancy. A followup MRI at one year post-surgery was normal.

\section{Discussion}

Expressive dystextia in this patient was coincident with headache and depression, both of which are common in the postpartum period [5] [20] [21]. Although expressive dystextia can be one component of expressive dysphasia, the latter is more often manifest verbally and as difficulty with written language [4]. Detection of dysphasia using text messages is obviously more challenging compared to conventional written composition since texting has fewer restrictions regarding grammar and punctuation [22], but our patient's text messages lacked syntax common to both written and text communication. Syntactic performance has been localized to the left inferior 


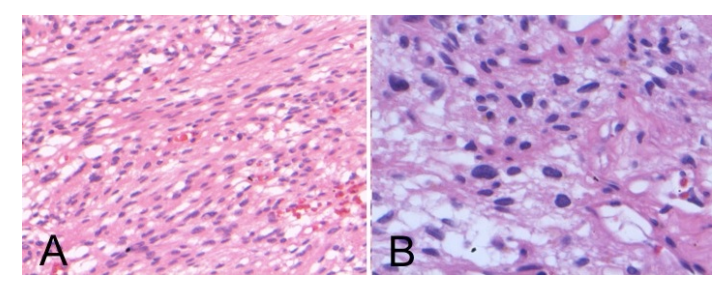

Figure 3. Intraoperative formalin-fixed WHO Grade I meningioma stained with hematoxylin and eosin, 200× magnification (A). No mitotic figures were observed, $400 \times$ magnification (B).

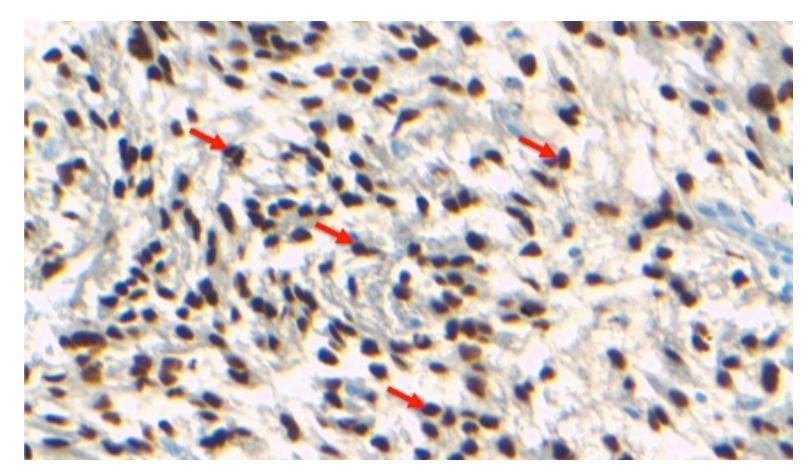

Figure 4. Moderate to strong nuclear progesterone receptor staining (red arrows) was evident in approximately $95 \%$ of cells, $400 \times$ magnification.

frontal gyrus and Brodmann area 45 [23], both of which could have been compressed in our patient due to her large right frontal mass and midline deviation. This expressive dystextia is distinct from the only other reported case of dystextia following left-sided stroke and hemiparesis in a poorly controlled type 2 diabetic that reduced the typing speed and accuracy of his text messages [1]. Following surgical resection of her meningioma, our patient rapidly recovered memory and language skills.

Long-term prognosis for WHO Grade 1 benign meningiomas is positive, particular in view of their expression of progesterone receptors and low mitotic rate [11]-[14] [24]. However, diagnosis of meningioma in our patient 18 years after previous thyroid cancer surgery at age 18 raises the possibility of a genetic link between the two neoplasms. Thyroid carcinoma and meningioma occasionally co-occur or appear as sequelae following radiation or chemotherapy, but this patient was not treated radiologically for her thyroid cancer [25]-[28]. A study of 1228 patients, 50 of whom had previous extracranial malignancies detected an epidemiological link between papillary carcinoma of the thyroid and meningioma [29]. In that series, none of the cancers was related to prior radiation exposure or a known preneoplastic syndrome such as multiple endocrine neoplasia (MEN). However, meningiomas have been added to the list of frequent neoplasms in multiple endocrine neoplasia type 1 linked by their loss of the MEN gene product, a putative tumor suppressor on chromosome 11q [30]. In that study, meningiomas appeared at an average of 18 years following the first neoplasm. However, genetic analysis was not undertaken on this patient.

A genetic etiology for meningioma tumorigenesis centers on chromosome 22q since about half of sporadic meningiomas exhibit monosomy, loss of heterozygosity or partial deletion of chromosome 22 [12]. Loss of expression of NF2 (Merlin) and DAL-1 tumor suppressors on 22q appear to be early events in the neoplastic transformation of meningioma as well as involvement in the pathogenesis of lung and breast cancers, tumors with elevated incidences in families of meningioma patients [28] [31] [32]. Sughrue et al. suggested a possible link between meningioma and thyroid carcinoma through upregulation of somatostatin receptors or through mutations of genes in the B-Raf/Merlin/NF2 pathway [29]. Other genes involved in the initial neoplastic transformation of meningiomas (meningioma gene MN1) may also be located on chromosome 22q. Progression to atypical or malignant meningiomas is associated with loss of progesterone receptor staining, an increased mitotic rate and with abnormalities of other chromosomes including loss of 1p [12] [22]. Such complex karyotypes 
are also present in radiation-associated meningiomas [12] [22]. Tumoral edema and angiogenesis, such as observed in our patient's meningioma, has been linked to production of vascular endothelial growth factor (VEGF) [12].

First-degree family history of meningioma increases relative risk for meningioma in younger patients by 5.4 times [28]. Family history of cervical, breast and lung cancer is also associated with meningioma, as is a personal history of uterine fibroid tumors, endometriosis and possibly breast cancer [28]. Since the presence of an early thyroid carcinoma in this meningioma patient is consistent with a genetic etiology, further genetic analysis could aid the elucidation of the molecular etiology of these benign progesterone receptor-positive neoplasms as well as provide guidance regarding surveillance for her and affected family members.

\section{Conclusion}

We present a postpartum patient with a progesterone-receptor-positive meningioma who developed increasing depression, headache and incomprehensible text messages (expressive dystextia). These symptoms were reversed following surgical resection of the meningioma. The expressive dystextia described in this case relates to loss of written syntactical skills and is distinct from other reported cases of dystextia that involve loss of motor skills following ischemic stroke. We speculate that this patient's meningioma may have a genetic basis since she also had a previous thyroid cancer, and these two cancers can share common genetic etiologies. Since text messaging is becoming one of the principal forms of communication in our society and requires both cognitive and motor skills, clinicians should be aware that dystextia may be the initial sign of significant neurologic pathology. We propose that an inquiry about altered text messaging frequency and comprehensibility should be a standard part of the neurologic evaluation.

\section{References}

[1] Cawood, T.J. (2006) Dystextia-A Sign of the Times? Irish Medical Journal, 99, 157.

[2] Ravi, A., Rao, V.R. and Klein, J.P. (2013) Dystextia: Acute Stroke in the Modern Age. Archives of Neurology, $70,404$. http://dx.doi.org/10.1001/jamaneurol.2013.604

[3] Burns, B. and Randall, M. (2013) "Dystextia”: Onset of Difficulty Writing Mobile Phone Texts Determines the Time of Acute Ischaemic Stroke Allowing Thrombolysis. Practical Neurology, 509. http://dx.doi.org/10.1136/practneurol-2013-000509

[4] Whitfield, P. and Jayathissa, S. (2011) Evolving Neurological Terminology in the $21^{\text {st }}$ Century: "Dystextia” Associated with Complex Migraine. Internal Medicine Journal, 41, 646. http://dx.doi.org/10.1111/j.1445-5994.2011.02507.x

[5] Chamoun, R., Krisht, K.M. and Couldwell, W.T. (2011) Incidental Meningiomas. Neurosurgical Focus, 31, 1-7. http://dx.doi.org/10.3171/2011.9.FOCUS11220

[6] Cohen-Gadol, A.A., Friedman, J.A., Friedman, J.D., Tubbs, R.S., Munis, J.R. and Meyer, F.B. (2009) Neurosurgical Management of Intracranial Lesions in the Pregnant Patient: A 36-Year Institutional Experience and Review of the Literature. Journal of Neurosurgery, 111, 1150-1157. http://dx.doi.org/10.3171/2009.3.JNS081160

[7] Cowppli-Bony, A., Bouvier, G., Rue, M., Loiseau, H., Vital, A., Lebailly, P., et al. (2011) Brain Tumors and Hormonal Factors: Review of the Epidemiological Literature. Cancer Causes and Control, 22, 697-714. http://dx.doi.org/10.1007/s10552-011-9742-7

[8] Kasper, E.M., Hess, P.E., Silasi, M., Lim, K.-H., Gray, J., Reddy, H., et al. (2010) A Pregnant Female with a Large Intracranial Mass: Reviewing the Evidence to Obtain Management Guidelines for Intracranial Meningiomas during Pregnancy. Surgical Neurology International, 1, 95. http://dx.doi.org/10.4103/2152-7806.74242

[9] Smith, J.S., Quiñones-Hinojosa, A., Harmon-Smith, M., Bollen, A. and McDermott, M.W. (2005) Sex Steroid and Growth Factor Profile of a Meningioma Associated with Pregnancy. Canadian Journal of Neurological Sciences, 32, 122-127.

[10] Wiemels, J., Wrensch, M. and Claus, E.B. (2010) Epidemiology and Etiology of Meningioma. Journal of Neuro-Oncology, 99, 307-314. http://dx.doi.org/10.1007/s11060-010-0386-3

[11] Choy, W., Kim, W., Nagasawa, D., Stramotas, S., Yew, A., Gopen, Q., et al. (2011) The Molecular Genetics and Tumor Pathogenesis of Meningiomas and the Future Directions of Meningioma Treatments. Neurosurgical Focus, 30, E6.

[12] Ragel, B.T. and Jensen, R.L. (2005) Molecular Genetics of Meningiomas. Neurosurgical Focus, 19 , E9. http://dx.doi.org/10.3171/foc.2005.19.5.10 
[13] Hsu, D.W., Efird, J.T. and Hedley-Whyte, E.T. (1997) Progesterone and Estrogen Receptors in Meningiomas: Prognostic Considerations. Journal of Neurosurgery, 86, 113-120. http://dx.doi.org/10.3171/jns.1997.86.1.0113

[14] Roser, F., Nakamura, M., Bellinzona, M., Rosahl, S.K., Ostertag, H. and Samii, M. (2004) The Prognostic Value of Progesterone Receptor Status in Meningiomas. Journal of Clinical Pathology, 57, 1033-1037. http://dx.doi.org/10.1136/jcp.2004.018333

[15] Gazzeri, R., Galarza, M. and Gazzeri, G. (2007) Growth of a Meningioma in a Transsexual Patient after EstrogenProgestin Therapy. New England Journal of Medicine, 357, 2411-2412. http://dx.doi.org/10.1056/NEJMc071938

[16] Vadivelu, S., Sharer, L. and Schulder, M. (2010) Regression of Multiple Intracranial Meningiomas After Cessation of Long-Term Progesterone Agonist Therapy. Journal of Neurosurgery, 112, 920-924. http://dx.doi.org/10.3171/2009.8.JNS09201

[17] Vernooij, M.W., Ikram, M.A., Tanghe, H.L., Vincent, A.J.P.E., Hofman, A., Krestin, G.P., et al. (2007) Incidental Findings on Brain MRI in the General Population. New England Journal of Medicine, 357, 1821-1828. http://dx.doi.org/10.1056/NEJMoa070972

[18] Baxter, D.S., Smith, P., Stewart, K. and Murphy, M. (2009) Clear Cell Meningioma Presenting as Rapidly Deteriorating Visual Field and Acuity During Pregnancy. Journal of Clinical Neurosciences, 16, 1502-1504. http://dx.doi.org/10.1016/j.jocn.2009.02.008

[19] Khong, S.Y., Leach, J. and Greenwood, C. (2007) Meningioma Mimicking Puerperal Psychosis. Obstetrics \& Gynecology, 109, 515-516. http://dx.doi.org/10.1097/01.AOG.0000226856.21741.3f

[20] Calado, S. and Viana-Baptista, M. (2006) Benign Cerebral Angiopathy; Postpartum Cerebral Angiopathy: Characteristics and Treatment. Current Treatment Options in Cardiovascular Medicine, 8, 201-212. http://dx.doi.org/10.1007/s11936-006-0013-y

[21] Singhal, A.B. and Bernstein, R.A. (2005) Postpartum Angiopathy and Other Cerebral Vasoconstriction Syndromes. Neurocritical Care, 3, 91-97. http://dx.doi.org/10.1385/NCC:3:1:091

[22] Coe, J.E.L. and Oakhill, J.V. (2011) “txtN is ez f u no h2 rd”: The Relation between Reading Ability and Text-Messaging Behaviour. Journal of Computer Assisted Learning, 27, 4-17. http://dx.doi.org/10.1111/j.1365-2729.2010.00404.x

[23] Tyler, L.K., Marsien-Wilson, W.D., Randall, B., Wright, P., Devereux, B.J., Zhuang, J., et al. (2011) Left Inferior Frontal Cortex and Syntax: Function, Structure and Behaviour in Patients with Left Hemisphere Damage. Brain, 134, 415-431. http://dx.doi.org/10.1093/brain/awq369

[24] Fewings, P.E., Battersby, R.D. and Timperley, W.R. (2000) Long-Term Follow Up of Progesterone Receptor Status in Benign Meningioma: A Prognostic Indicator of Recurrence? Journal of Neurosurgery, 92, 401-405. http://dx.doi.org/10.3171/jns.2000.92.3.0401

[25] Barg, E., Godzinski, J., Wikiera, B., Gtab, E. and Jelen, M. (2009) Papillary Thyroid Carcinoma and Meningioma in 17-Year-Old Boy-A Late Effect of Acute Lymphoblastic Leukemia Treatment or a New Disease? Case Report and Literature Review. Pediatric Endocrinology, Diabetes \& Metabolism, 15, 51-54.

[26] Brassesco, M.S., Valera, E.T., Neder, L., Pezuk, J.A., Oliviera, R.S., Scrideli, C.A., et al. (2012) Cytogenetic Findings in Pediatric Radiation-Induced Atypical Meningioma after Treatment of Medulloblastoma: Case Report and Review of the Literature. Journal of Neuro-Oncology, 110, 397-402. http://dx.doi.org/10.1007/s11060-012-0982-5

[27] Chaturvedi, S., Gupta, S. and Kumari, R. (2010) Meningioma with Metastasis from Follicular Carcinoma Thyroid. Indian Journal of Pathology \& Microbiology, 53, 316-318. http://dx.doi.org/10.4103/0377-4929.64298

[28] Claus, E.B., Calvocoressi, L., Bondy, M.L., Schildkraut, J.M., Wiemels, J.L. and Wrensch, M. (2011) Family and Personal Medical History and Risk of Meningioma. Journal of Neurosurgery, 115, 1072-1077. http://dx.doi.org/10.3171/2011.6.JNS11129

[29] Sughrue, M.E., Kane, A.J., Shangari, G., Parsa, A.T., Berger, M.S. and McDermott, M.W. (2010) Prevalence of Previous Extracranial Malignancies in a Series of 1228 Patients Presenting with Meningioma. Journal of Neurosurgery, 113, 1115-1121. http://dx.doi.org/10.3171/2010.3.JNS091975

[30] Asgharian, B., Chen, Y.-J., Patronas, N.J., Peghini, P.L., Reynolds, J.C., Vortmeyer, A., et al. (2004) Meningiomas May be a Component Tumor of Multiple Endocrine Neoplasia Type 1. Clinical Cancer Research, 10, 869-880. http://dx.doi.org/10.1158/1078-0432.CCR-0938-3

[31] Gutmann, D.H., Donahoe, J., Perry, A., Lemke, N., Gorse, K., Kittiniyou, K., et al. (2000) Loss of DAL-1, a Protein 4.1-Related Tumor Suppressor, Is an Important Early Event in the Pathogenesis of Meningiomas. Human Molecular Genetics, 9, 1495-1500. http://dx.doi.org/10.1093/hmg/9.10.1495

[32] Perry, A., Can, D.X., Scheithauer, B.W., Swanson, P.E., Lohse, C.M., Newsham, I.F., et al. (2000) Merlin, DAL-1, and Progesterone Receptor Expression in Clinicopathologic Subsets of Meningioma: A Correlative Immunohistochemical Study of 175 Cases. Journal of Neuropathology \& Experimental Neurology, 59, 782-879. 


\section{List of Abbreviations}

B-Raf: Serine/Threonine Protein Kinase-B-Raf

CAM5.2: Anti-Low Molecular Weight Cytokeratin Antibody

CT: Computed Tomography

DAL-1: Erythrocyte Membrane Protein Band 4.1-Like 3 Gene

EMA: Epithelial Membrane Antigen

GFAP: Glial Fibrillary Acidic Protein

Ki-67: Antigen KI-67

MEN: Multiple Endocrine Neoplasia Gene

MN1: Meningioma (Disrupted in Balanced Translocation 1) Gene

MRI: Magnetic Resonance Imaging

NF2: Neurofibromatosis 2 Tumor Suppressor Gene

TTF-1: Thyroid Transcription Factor-1

VEGF: Vascular Endothelial Growth Factor

WHO: World Health Organization 